\title{
EXTENSION SETS
}

\author{
BY \\ A. D. WALLACE
}

1. Historical introduction. In a symposium lecture in $1932 \mathrm{R}$. L. Wilder $[10]\left({ }^{1}\right)$, in discussing the structure of continua, indicated the possibility of extending G. T. Whyburn's cyclic element theory by making use of combinatorial methods. The notion of an " $n$th order cyclic element" was developed in a paper published by Whyburn [8] some two years later. In this paper Wilder's suggestion will be modified in replacing "combinatorial methods" by "analytic methods." Considerable dependence will be placed on the ideas and techniques formulated by C. Borsuk, S. Eilenberg and W. Hurewicz. It is perhaps worthy of remark that Wilder does not refer to the third "branch" of topology, analytic topology, in this lecture though it played a prominent part in another of his lectures given about six years later [11].

It will be recalled that an $A$-set is a retract of its containing space, so that, guided by Borsuk's result characterizing absolute retracts as those spaces which have the extension property, we are led to the notion of an extension set which plays the part of an $A$-set. However an extension set is a relative and not an absolute invariant because, by necessity, it must be intimately related to its containing space.

Section two will be devoted to results of a preliminary nature, mainly existence theorems. In the next section we consider sets that are "trivial" in dimension $n$; more exactly - sets which are hereditary relative to the property of admitting no essential transformations into the $n$-sphere. For separable spaces some of the results here are immediate consequences of well known theorems of dimension theory. The notion of an extension set is introduced in the fourth section. These sets have many of the properties possessed by $A$-sets in a locally connected continuum. Thus the intersection of any family of extension sets is again an extension set. Also our "cyclic elements" are extension sets. With the addition of a separation axiom it is shown that an extension set in one dimension is also an extension set of any higher dimension. The notion of a general "endelement" is introduced and also shown to be an extension set. The study of these sets is continued in section five where other results are formulated. Scattered through the paper are theorems concerning "continua of order $n$ " and "endelements of order $n$. "

While no direct use will be made of their results it is necessary to call attention to the work of several mathematicians who have studied and gen-

Presented to the Society, June 24, 1945; received by the editors May 17, 1945.

( $\left.{ }^{1}\right)$ Numbers in brackets refer to the Bibliography at the end of the paper. 
eralized the cyclic element theory: W. L. Ayres, V. W. Adkisson, G. E. Albert, D. W. Hall, F. B. Jones, J. L. Kelley, C. Kuratowski, Saunders MacLane, R. L. Moore, T. Radó, P. V. Reichelderfer, Hassler Whitney and J. W. T. Youngs. References to their papers will be found in [7] and [9].

References should also be made to papers by J. Rozanska [6] and C. Borsuk [12] containing concepts and results which are related to ideas and theorems formulated here.

I am glad to acknowledge that my interest in non-separable spaces and in homotopy theory springs from many discussions with S. Lefschetz.

2. Mathematical introduction. While many of our results are valid in a more general situation an optimum degree of generality will consistently be attained if we assume that $H$ is a compact Hausdorff space. Here compact replaces the more unwieldy "bicompact." In the further interests of verbal simplicity we use subspace for "closed subset of $H$."

The space $H$ is, of course, normal. We introduce the additional separation axiom as follows: A topological space $F$ is said to be of type $V$ if for any pair of closed subsets $R_{1}$ and $R_{2}$ there exists a decomposition of $F$ into closed sets $F_{1}$ and $F_{2}$ containing $R_{1}$ and $R_{2}$ such that $F_{1} \cdot F_{2} \cdot\left(R_{1}+R_{2}\right)=R_{1} \cdot R_{2}$. We are unable to give a reference to the occurrence of this condition as an axiom though it is a well known property of metric spaces. In a later paper it will be necessary to consider spaces in which each subset has the above property. When $H$ is assumed to be of type $V$ attention will be called to this postulate.

We denote by $S$ a compact nondegenerate Hausdorff space which has the neighborhood extension property: Any mapping of a closed set $A$ of a normal space into $S$ can be extended to a mapping of a neighborhood $U$ of $A$ into $S$. When the spaces involved are separable metric then the neighborhood extension property is equivalent to the property of being an absolute neighborhood retract as Borsuk $[4$, p. 60$]$ has shown.

The words "mapping" and "transformation" are used in the sense of "continuous correspondence" and "into" and "onto" have their usual meaning. Two transformations $f, g$ into $S$ are said to be homotopic (in symbols $f \sim g$ ) if there is a mapping $h$ defined for all points $x$ under consideration and all $t \in(01)$ such that $h(x, 0)=f(x)$ and $h(x, 1)=g(x)$. The values of $h$ are assumed to lie always in $S$. No confusion is to be feared concerning the symbol $\sim$ since we do not use homology in this paper. In most of our results $S$ will be taken to be an $n$-sphere $S_{n}$. We need the following result of Borsuk as extended by Dowker [3, p. 86]:

(2.1) If $f$ maps $H$ into $S$ and $g$ maps the subspace $X$ into $S$ and $f \sim g$ on $X$ then $g$ has an extension $\bar{g}: H \rightarrow S$ such that $f \sim \bar{g}$ on $H$.

A mapping $f$ of a topological space $F$ into $S$ is said to be inessential if it is homotopic to a mapping $g$ such that $g(F)$ is a proper subset of $S$. Also $f$ is null-homotopic (in symbols, $f \sim 0$ ) if $f$ is homotopic to a transformation $g$ 
for which $g(F)$ is a point of $S$. It is obvious that an inessential mapping into an $n$-sphere is null-homotopic.

(2.2) If $f$ and $g$ are mappings of $H$ into $S$ and $f \sim g$ on the subspace $X$ then $f \sim g$ on some open set containing $X$.

Proof. Let $h$ be a transformation of $X \times(01)$ into $S$ such that $h(x, 0)=f(x)$ and $h(x, 1)=g(x)$. We may extend the definition of $h$ by putting $h=f$ on $X \times(0)$ and $h=g$ on $X \times(1)$. Then $K=H \times(0)+X \times(01)+H \times(1)$ is a closed set in the cartesian product of $H$ and (01). In virtue of the fact that $S$ has the neighborhood extension property, we may suppose that $h$ has been extended to a mapping of a neighborhood $U$ of $K$. In view of a lemma of Dowker's $[3$, p. 86] there is an open set $V$ containing $X$ and for which we have the inclusion $V \times(01) \subset U$. Then clearly $f$ and $g$ are homotopic on the set $V$.

(2.3) If $f$ is a transformation of a subspace $Y$ into $S$ and $f \sim 0$ on the subspace $X \subset Y$ then $f \sim 0$ on an open subset of $Y$ containing $X$.

This follows from (2.2) and is a generalization of a result due to $\mathrm{S}$. Eilenberg $[9$, p. 221].

The term ordered, as applied to a family of subspaces, will mean that, of any pair, one is a subset of the other.

A property $P$ admissible for subspaces will be termed inductive if, when each of an ordered family of subspaces has property $P$, then their intersection also has property $P$. Many writers have formulated results closely related to the following which we call simply the "Brouwer induction theorem." References will be found in $[7$, p. 488].

(2.4) If $P$ is an inductive property and the subspace $X$ has $P$ then there exists a subspace contained in $X$ and minimal relative to having property $P$.

A mapping $f$ of the subspace $X$ into $S$ is said to be irreducibly essential on $X$ provided that $f$ is essential on $X$ but inessential on any subspace properly contained in $X$.

(2.5) An essential transformation of $H$ into $S$ is irreducibly essential on some subspace.

Proof. If we refer to (2.4) it is enough to show that, if $P$ is the property of being a subspace on which the mapping $f: H \rightarrow S$ is essential, then $P$ is an inductive property. To this end let $[X]$ be an ordered family of subspaces on each of which $f$ is essential and let $X_{0}$ be their intersection. If $f$ is inessential on $X_{0}$ then $f$ is homotopic to a transformation $g$ of $X_{0}$ into $S$ such that $g\left(X_{0}\right)$ is a proper subset of $S$. Since $S$ has the neighborhood extension property we may suppose that $g$ has already been extended to an open set $U$ which contains $X_{0}$. Moreover, $H$ is normal. Hence, because we can find a neighborhood 
of $X_{0}$ whose closure is contained in $U$, we may assume that $g$ is defined on $\bar{U}$. Then, by (2.2), since $f \sim g$ on $X_{0}$, there exists a neighborhood $V$ of $X_{0}$ contained in $U$ such that $f$ is homotopic to $g$ on $V$. In virtue of the fact that $S$ is a Hausdorff space the set $g\left(X_{0}\right)$ is closed and in virtue of the normality of $S$ there is an open set $W$ which contains $g\left(X_{0}\right)$ and such that $S-W$ is not vacuous. For each $x \in X_{0}$ let $P_{x}$ be a neighborhood of $x$ with $P_{x} \subset V$ and $g\left(P_{x}\right) \subset W$. Let $Q$ be the union of all the sets $P_{x}$. Then $Q$ is an open set containing $X_{0}, Q \subset V$ and $g(Q) \subset W$. The open sets $[H-X]$ cover the closed set $H-Q$ and hence from the compactness of $H$ and the fact that [X] is an ordered family we conclude that $H-Q$ is contained in some single set $H-X^{\prime}$. Thus $X^{\prime} \subset Q$. But $f \sim g$ on $V$ and so on $X^{\prime}$ and clearly $g\left(X^{\prime}\right)$ is a proper subset of $S$. This contradiction completes the proof.

This is a generalization of a result of G. T. Whyburn's [9, p. 222]. The next definition (though not the nomenclature) and the result which follows are due to W. Hurewicz $[3$, p. 94]. Let $f: X \rightarrow S$ and let $Y$ be a subspace such that $f$ admits an extension to any closed subset of $Y$ but not to $Y$ itself. Then $Y$ is said to be an essential membrane for $f$. Only trivial changes are necessary to modify Hurewicz's proof so that we get:

(2.6) Any mapping of a subset $X$ of $H$ into $S$ which cannot be extended to a mapping of $H$ into $S$ admits an essential membrane.

The next result is due to $S$. Eilenberg [2, p. 164].

(2.7) If the subspace $X$ admits an irreducibly essential mapping into an $n$-sphere then (a) if $n=0, X$ is $a$-sphere and (b) if $n$ is positive then $X$ is a continuum.

3. Connectivity and the sets $T_{S}$. In this section we shall be concerned with a generalization of the notion of connectedness and sets that are "trivial" relative to mappings into $S$. Most of our results follow rather easily from classical theorems if we admit that $H$ is metric.

A topological space $R$ is said to be $S$-connected provided that every mapping of $R$ into $S$ is inessential on each compact subset of $R$. By an $S$-continuum (or a $C_{S}$ ) is meant a compact $S$-connected space.

Here and later we shall be interested mainly in the situation in which $S$ is an $n$-sphere. In this case we write $n$-connected, $n$-continuum and $C_{n}$ respectively. It is easy to see that a 0 -connected set is connected in the usual sense and conversely, under relatively mild conditions. Also if $H$ is metric and locally connected than it is 1-connected if and only if it is unicoherent, a fact first proved by Borsuk. A more general situation was considered by Eilenberg (see [9]).

(3.1) The property of being an S-connected subspace of $H$ is inductive.

Proof. Let $[X]$ be an ordered family of $S$-continua of $H$ and let $X_{0}$ denote 
the intersection of these sets. Let $f: X_{0} \rightarrow S$. We may assume that $f$ has been extended to a neighborhood $U$ of $X_{0}$. From the compactness of $H$ and the fact that the collection $[X]$ is ordered we conclude that some $X^{\prime}$ of $[X]$ is contained in $U$. In virtue of the fact that $X^{\prime}$ is a $C_{S}$ we see that $f$ is inessential on $X^{\prime}$ and hence on $X$.

For $S=S_{0}$ this result is classical. For $S=S_{1}$ see Whyburn [9, p. 222]. From (3.1) and the Brouwer induction theorem we conclude, for example, that if $H$ is a $C_{S}$, then any subspace admits an $S$-continuum irreducible about it.

A compact Hausdorff space is said to be a $T_{S}$ provided that every one of its closed sets is a $C_{S}$. In other words a $T_{S}$ is hereditarily a $C_{S}$. Clearly a closed subset of $H$ is a $T_{0}$ if and only if it is a point.

(3.2) Any $T_{n}$ is a $T_{n+1}$.

Proof. Let $Y$ be a $T_{n}$ and let $X$ be a closed subset of $Y$. Suppose that $f$ is a mapping of $X$ into $S_{n+1}: y_{1}^{2}+\cdots+y_{n+2}{ }^{2}=1$. Decompose $S_{n+1}$ into two $(n+1)$-cells $A_{1}$ and $A_{2}$ defined by $y_{n+2} \geqq 0$ and $y_{n+2} \leqq 0$ respectively. Clearly $f(X)$ covers $S_{n+1}$ or else $f$ is null-homotopic. Let $B_{i}=f^{-1}\left(A_{i}\right), C=B_{1} \cdot B_{2}$, so that $C$ is the inverse under $f$ of the $n$-sphere $D=A_{1} \cdot A_{2}$. Let $g=f \mid C$. We know that $g$ is not essential. Then there exists a mapping $g^{\prime}: C \times(01) \rightarrow D$ such that $g^{\prime}(x, 0)=g(x)$ and $g^{\prime}(x, 1)=\bar{y}$, a point of $D$. We may extend $g^{\prime}$ (retaining the notation) to $B_{1} \times(0)+B_{1} \times(1)$ by setting $g^{\prime}(x, 0)=g(x), g^{\prime}(x, 1)=\bar{y}$ on $B_{1}$. Then $g^{\prime}$ transforms $B_{1} \times(0)+C \times(01)+B_{1} \times(1)$ into the $(n+1)$-cell $A_{1}$ and so may be extended to a mapping $h_{1}$ of $B_{1} \times(01)$ into $A_{1}$ by Tietze's theorem [3, p. 82]. Similarly we secure a mapping $h_{2}$ of $B_{2} \times(01)$ such that $h_{1}=h_{2}$ on the common part of $B_{1} \times(01)$ and $B_{2} \times(01)$. We may then combine $h_{1}$ and $h_{2}$ to get a transformation $h$ of $X \times(01)$ into $S_{n+1}$ such that $h(x, 0)=f(x)$ and $h(x, 1)=\bar{y}$ for all $x \in X$. Accordingly $f$ is not essential. Thus $Y$ is a $T_{n+1}$.

A subspace will be termed a $B_{S}$ provided that it is not cut by any $T_{S}$ and is maximal relative to this property. For this notion and (3.3) see G. T. Whyburn [8].

(3.3) If $X$ is a subspace not cut by any $T_{S}$ then $X$ is contained in a $B_{S}$. Each $B_{S}$ is a 0-continuum and the intersection of any distinct pair of them is a set $T_{S}$.

(3.4) For any $B_{n}$ there exists a sequence $B_{0} \supset B_{1} \supset \cdots \supset B_{n}$.

This result follows at once from (3.2).

(3.5) If the subspace $Y$ is the union of a countable collection of sets $T_{n}$ then $Y$ is $a T_{n+1}$.

Proof. Let $X$ be a subspace contained in $Y$ and let $f: X \rightarrow S_{n+1}$. Since any subspace contained in a $T_{n}$ is also a $T_{n}$ we may set $X=X_{1}+X_{2}+X_{3}+\cdots$, where each $X_{i}$ is a $T_{n}$. We may assume that $f$ has been extended to a neigh- 
borhood $U$ of $X$ in virtue of the fact that, as an ANR, $X$ has the neighborhood extension property. There is a neighborhood $U_{1}$ such that $Y_{1}=X_{1} \subset U_{1} \subset \bar{U}_{1}$ $\subset U$ such that $f \sim 0$ on $\bar{U}_{1}$, by (2.3) and (3.2). Let $Y_{2}=\bar{U}_{1}+X_{2}$. We may assume that $f\left(Y_{2}\right)$ covers $S_{n+1}$ since otherwise $f$ would be null-homotopic on $Y_{2}$. Let $S_{n+1}$ be given by $y_{1}^{2}+\cdots+y_{n+2}{ }^{2}=1$ and define $g$ as the transformation which takes $\bar{U}_{1}$ into the point $\bar{y}=(0,0, \cdots, 0,1)$. Now $g$ is null-homotopic on $\bar{U}_{1}$ so that by the reflexivity of homotopy we have $f \sim g$ on this set. Hence by (2.1) we may extend $g$ to a mapping $h: Y_{2} \rightarrow S_{n+1}$ such that $f \sim g$ on $Y_{2}$. Let $S_{n}$ be defined as the subset of $S_{n+1}$ on which $y_{n+2}=0$. Then $h^{-1}\left(S_{n}\right)$ lies wholly in $X_{2}-\bar{U}_{1}$. Now if $f$ were essential on $Y_{2}$ then the transformation $h$ regarded as a mapping of $h^{-1}\left(S_{n}\right)$ into $S_{n}$ would be essential by the argument given in the proof of (3.2). This is impossible since $h^{-1}\left(S_{n}\right)$ lies in the $T_{n}$, $X_{2}-\bar{U}_{1}$. Hence we see that $f$ is null-homotopic on $Y_{2}$. Accordingly there is a neighborhood $U_{2}$ of $Y_{2}$ such that $\bar{U}_{2}$ is contained in $U$ and $f$ is inessential on $U_{2}$. In this way we secure for each integer $i$ a neighborhood $U_{i}$ for which

$$
Y_{i}=\bar{U}_{i-1}+X_{i} \subset U_{i} \text { and } f \sim 0 \text { on } Y_{i}=\bar{U}_{i}+X_{i+1} \subset U \text {. }
$$

Since the open sets $U_{i}$ cover the compact set $X$ and in addition form an increasing family it follows that $X$ lies in some set $U_{j}$. Thus $f$ is null-homotopic on $X$ and so $X$ is a $T_{n+1}$.

We terminate this section with some additional theorems on $n$-continua.

\section{(3.6) $A$ retract of $a C_{n}$ is a $C_{n}$.}

Proof. Suppose that $H$ is an $n$-continuum and let $r: H \rightarrow X$ be a retraction. Then $X$ is a subspace so let $f: X \rightarrow S_{n}$ and set $g=f r$ so that $g$ transforms $H$ in to the $n$-sphere. Then $g$ is inessential. But $g \mid X=f$ so that $f$ is also inessential.

It is easy to see that a Tychonoff cube is a $C_{n}$ for any $n$. Indeed it is clear that we have:

(3.7) If a subspace can be deformed over itself to a point then it is a $C_{n}$.

It is also clear that an $n$-cell is a $T_{n}$. For it is a $C_{n}$ and a mapping of any of its closed sets into $S_{n}$ can be extended by a theorem of Hurewicz [3, p. 83].

Also it is of interest to observe the difference between a $C_{n}$ and a $\gamma^{n}$-continuum [8]. In virtue of a result of H. Hopf [13] it is clear that $S_{3}$ is not a $C_{2}$ while it is a $\gamma^{2}$-continuum.

We say that a subspace $X$ of $H$ is a divisor of $H$ if $H \times(0)+X \times(01)+H$ $\times(1)$ is a retract of $H \times(01)$.

(3.8) If the subspace $X$ is both a divisor of $H$ and a $C_{n}$ then $H$ is a $C_{n}$.

Proof. Let $f$ be a mapping of $H$ into the $n$-sphere and let $g=f \mid X$. Then $g$ is null-homotopic and so there exists a transformation $h: X \times(01) \rightarrow S_{n}$ such that $h(x, 0)=g(x)$ and $h(x, 1)=\bar{y} \in S_{n}$. We may extend $h$ (conserving the notation) to a mapping of $K=H \times(0)+X \times(01)+H \times(1)$ into the $n$-sphere by letting 
$h=f$ on $H \times(0)$ and $h=\bar{y}$ on $H \times(1)$. Let $r$ retract $H \times(01)$ into $K$. We may then set $k=h r$ so that $k$ maps $H \times(01)$ into $S_{n}$ and $k=h$ on $H \times(0)$ and $k=\bar{y}$ on $H \times(1)$.

We return to the notion of a divisor in a later paper. The concept is a modification of one introduced by Lusternik and Schnirelmann [5, p. 40]. The result (3.8) is introduced here since, in its contra-positive form, it is helpful in examples.

(3.9) If $f: X \rightarrow S_{n}$ is irreducibly essential on the subspace $X$ and $Y$ is an essential membrane for $f$ then $Y$ is a $C_{0}$.

Proof. Let $n$ be positive and suppose that $Y=P+Q$ where $P$ and $Q$ are disjoint closed subsets of $Y$. In virtue of (2.7) we may suppose that $X \subset P$ and then let $\bar{f}$ be an extension of $f$ mapping $P$ into $S_{n}$. It is then trivial that $\bar{f}$ admits an extension to $P+Q$, a contradiction. A similar argument is valid if $n=0$.

4. Extension sets. By an extension set of order $n$ of $H$ (or a $J_{n}$ ) will be meant a subspace $M$ such that for each closed set $X$ each mapping of $M \cdot X$ into the $n$-sphere can be extended to a mapping of $X$ into the $n$-sphere. Here we shall prove, for example, that the intersection of extension sets is an extension set and that the sets $B_{n}$ are extension sets. With the assumption that $H$ is of type $V$ we are able to show that a $J_{n}$ is a $J_{n+1}$. It is clear that an extension set is a topological invariant of $H$ and that any point of $H$ is an extension set. Many of our results are generalizations of well known results of G. T. Whyburn and W. L. Ayres (see [9]).

\section{(4.1) The space $H$ and each $T_{n}$-set is an extension set of order $n$.}

Proof. If $M$ is a $T_{n}$ and $X$ is a subspace then any transformation $f$ of $M \cdot X$ into $S_{n}$ is inessential and hence by (2.1) may be extended to a mapping of $X$ into $S_{n}$ since the trivial mapping may always be extended.

(4.2) If $[M]$ is an arbitrary collection of sets $J_{n}$ then their intersection is also a $J_{n}$.

Proof. Let $X$ be a subspace and $f$ a transformation of $M_{0} \cdot X$ into the $n$-sphere, where $M_{0}$ is the intersection of all the sets in $[M]$. We may extend $f$ (keeping the same notation) to a neighborhood $U$ of $M_{0} \cdot X$ in virtue of the fact that $S_{n}$ has the neighborhood extension property. In view of the compactness of $H$ and the fact that the sets $[M]$ are closed it follows that there exists (by the Borel theorem) a finite family $M_{1}, M_{2}, \cdots, M_{p}$ such that $M_{1} \cdot M_{2} \cdots M_{p} \cdot X \subset U$. Let $g_{1}=f \mid M_{1} \cdot M_{2} \cdots M_{p} \cdot X$. Now $M_{1}$ is an extension set and so $g_{1}$ may be extended to a mapping $g_{2}: M_{2} \cdots M_{p} \cdot X \rightarrow S_{n}$. Similarly $g_{2}$ may be extended to a mapping $g_{3}$ of the set $M_{3} \cdots M_{p} \cdot X$ into the $n$-sphere. Continuing in this way we arrive finally at a mapping $g$ of $X$ 
into $S_{n}$ which is an extension of $f$. Accordingly we have shown that $M_{0}$ is an extension set of order $n$.

Let $M$ be a $J_{0}$ lying in the Peano space $H$ and let $C$ be an arc having only its end points $a$ and $b$ in $M$. Now $S_{0}$ consists of the point -1 and the point +1 of the real line. Define $f(a)=-1$ and $f(b)=+1$. Then obviously $f$ cannot be extended to $C$ since $C$ is connected.

At this point it is perhaps well to give some examples. Let $L$ be the interval from $(0,-1)$ to $(0,1)$ in the plane and let $W$ be the curve $y=\sin x^{-1}$ for $0<x \leqq 1$. Then $H$ is the union of the sets $W$ and $L$. The set $L$ is a $J_{n}$ for all non-negative integers $n$.

As another example let $L$ be the unit interval and $C$ the Cantor set on $L$. At each point $c$ of $C$ let $I(c)$ be an interval of unit length erected perpendicular to $L$ and above $L$. Finally let $H$ be the Cartesian product of (01) with the set composed of $L$ and all the sets $I(c)$. Then $E \times(01)$ is a $J_{n}$ for all positive integers $n$. It is easy to see that $L \times(01)$ is not a $J_{0}$.

The next example I owe to J. W. Tukey. Let $C_{1}$ and $C_{2}$ be concentric 1 -spheres of radii 1 and 2 respectively. Let $H$ be the closed ring they determine. For each point $z$ of $C_{1}$ let $R(z)$ be that part of the ray through the center of $C_{1}$ which lies in $H$. We set up a topology in $H$ as follows: (a) If $z$ is a point of $C_{1}$ then a neighborhood of $z$ will consist of an open arc of $C_{1}$ containing $z$ together with all sets $R\left(z^{\prime}\right)$ for all $z^{\prime}$ in the open arc with the exception that on $R(z)$ there may have been deleted any closed interval not containing the point $z$. (b) If $x$ is a point of $R(z)$ distinct from $z$ then a neighborhood of $x$ will be an open interval of $R(z)$ containing $x$. Then $H$ is a compact connected locally connected Hausdorff space but is not separable. The set $C_{1}$ is a $J_{n}$ for all positive $n$ but is not a $J_{0}$.

(4.3) If $M$ is $a J_{n}$ and $X$ is $a C_{n}$ then $M \cdot X$ is $a C_{n}$.

Proof. Let $f$ map $M \cdot X$ into the $n$-sphere and let $\bar{f}$ be an extension of $f$ transforming $X$ into the $n$-sphere. Since $\bar{f}$ is inessential on $X$ it is manifestly inessential on $X$. But $f=\bar{f} \mid M \cdot X$ and so $f$ is inessential. Accordingly $M \cdot X$ is a $C_{n}$.

This result carries with it the conclusion that if $H$ is a $C_{n}$ then so also is each $J_{n}$. For any set $A$ contained in $H$ let $\Delta_{n}(A)$ be the intersection of all sets $J_{n}$ which contain $A$. Since $H$ is a $J_{n}$ the set $\Delta_{n}(A)$ is always defined. By (4.2) it follows that:

(4.4) For any set $A \subset H$ the set $\Delta_{n}(A)$ is a $J_{n}$.

It is not hard to see that if $H$ is a Peano space then the $J_{0}$-sets and $A$-sets are identical as are $B_{0}$-sets and cyclic elements. If we omit the condition of local connectedness then each $A$-set is a $J_{0}$ but the converse is false as may be seen by simple examples. (In this connection see (5.4).) Also here the $B_{0}$-sets are identical with the $E_{0}$-sets. 
We wish to show that any $B_{n}$ is a $J_{n}$. We begin with:

(4.5) If the subspace $X$ is not cut by any $T_{n}$ then $\Delta_{n}(X)$ also has this property.

Proof. Let $Z$ be a $T_{n}$ cutting $\Delta_{n}(X)=M$ so that $M-Z=U+V$ where $U$ and $V$ are mutually separated. Now $X$ is not a subset of $Z$ since otherwise $X$ would be a $T_{n}$ and certainly some closed subset of $X$ cuts $X$. We may then suppose that $X-Z \subset U$ so that $U$ is not void. Let $K$ be a subspace, $N=U+Z$ and let $f$ be a mapping of $N \cdot K$ into the $n$-sphere. Now $K \cdot M$ $=K \cdot N+K \cdot(V+Z)$ and we have $(K \cdot N) \cdot K \cdot(V+Z)=K \cdot Z$ and this set is a $T_{n}$. Thus $f \mid K \cdot Z$ is null-homotopic and so by (2.1) may be extended to a mapping of $K \cdot(V+Z)$ into $S_{n}$. We thus arrive at an extension of $f, \bar{f}: K \cdot M$ $\rightarrow S_{n}$. From (4.4) it follows that $\bar{f}$ may be extended to a transformation of $K$ into the $n$-sphere. Thus $N$ is a $J_{n}$ which contains $X$ and does not meet $V$. Accordingly $V=0$ and the proof is complete.

(4.6) Each set $B_{n}$ is also a set $J_{n}$.

Proof. Let $B$ be a $B_{n}$ so that $B$ is not cut by any $T_{n}$ and is maximal relative to this property. Then $\Delta_{n}(B)$ is not cut by any $T_{n}$ and $B C \Delta_{n}(B)$. Hence $B=\Delta_{n}(B)$ and so is a $J_{n}$ by (4.4).

At this point it seems worthwhile to comment on the fact that, although defined by quite different devices, the sets $B_{n}$ are in a sense the nontrivial minimal sets of type $J_{n}$. Thus (4.6) lends a certain uniformity to the structural theory we develop.

(4.7) If $H$ is a space of type $V$ then each $J_{n}$ is a $J_{n+1}$.

Proof. Let $M$ be an extension set of order $n, Y$ a subspace and $f$ a transformation of $M \cdot Y$ into the $(n+1)$-sphere. As usual we suppose that $S_{n+1}$ is given by $y_{1}{ }^{2}+\cdots+y_{n+2}{ }^{2}=1$ so that the sets $A_{1}$ and $A_{2}$ defined by $y_{n+2} \geqq 0$ and $y_{n+2} \leqq 0$ are $(n+1)$-cells meeting in an $S_{n}$. If $f(M \cdot Y)$ does not cover $S_{n+1}$ then $f$ is null-homotopic and so may be extended to $Y$ by (2.1). We may thus suppose that $f(M \cdot Y)=S_{n+1}$ and let $Q_{i}$ be the inverse of $A_{i}$ under $f$ so that $M \cdot Y=Q_{1}+Q_{2}$. Let $R=Q_{1} \cdot Q_{2}$. Since $H$ is of type $V$ (see the first part of section two) we may write $H=H_{1}+H_{2}$ where $Q_{i} \subset H_{i}, H_{1} \cdot H_{2} \cdot M \cdot Y=R$, with $H_{1}$ and $H_{2}$ closed. Put $Y_{i}=H_{i} \cdot Y$ so that we have $Q_{i} \subset Y_{i}, Y_{1} \cdot Y_{2} \cdot M \cdot Y=R$ and $Y=Y_{1}+Y_{2}$. Now let $g=f \mid R$ so that $g$ maps $R$ into $S_{n}$. Since $M$ is a $J_{n}$ we may exterid $g$ to $\bar{g}: Y_{1} \cdot Y_{2} \rightarrow S_{n}$. Let $f_{1}=f \mid Q_{1}$ and $f_{1}=\bar{g} \mid Y_{1} \cdot Y_{2}$ so that $f_{1}$ is a mapping of $Q_{1}+Y_{1} \cdot Y_{2}$ into $A_{1}$. By Tietze's extension theorem there is an extension of $f_{1}, h_{1}: Y_{1} \rightarrow A_{1}$. Similarly we construct a transformation $h_{2}: Y_{2} \rightarrow A_{2}$ for which we have $h_{1}=h_{2}$ on $Y_{1} \cdot Y_{2}$. If we combine $h_{1}$ and $h_{2}$ we secure a mapping $h: Y \rightarrow S_{n}$ such that $h \mid M \cdot Y=f$. Thus $M$ is a $J_{n+1}$.

(4.8) If $H$ is of type $V$ and $A \subset H$ then

$$
\Delta_{0}(A) \supset \Delta_{1}(A) \supset \cdots \supset A \text {. }
$$


The proof of (4.8) is immediate from (4.7). This result should be compared with (3.4).

(4.9) Let $M$ be a $J_{n}$ and let $f: X \rightarrow S_{n}$ where $X$ is a closed subset of $M$. If $N$ is an essential membrane for $f$ then $N \subset M$.

Proof. By definition $f$ can be extended to any proper subspace of $N$. If $N$ is not contained in $M$ then $f$ can be extended to $M \cdot N$ and then to $N$ since $M$ is an extension set.

In an entirely analogous manner it is possible to prove that:

(4.10) If $M$ is a $J_{n}, X$ a subset of $M$ and $N$ a $C_{n}$ irreducible about $X$, then $N \subset M$.

Let $P$ be a property admissible for subspaces. A closed subset $X$ of $H$ will be termed a $P$-endelement provided that for each neighborhood $U$ of $X$ there is a neighborhood $V$ of $X$ contained in $U$ such that $F(V)=\bar{V}-V$ has property $P$. If $X$ is a point we say that it is a $P$-endpoint.

(4.11) For any property $P$ the property of being a P-endelement is inductive.

The proof of (4.11) is immediate in view of the compactness of $H$.

(4.12) Any $T_{n}$-endelement of $H$ is a $J_{n}$.

Proof. Let $M$ be a $T_{n}$-endelement, $Y$ a subspace and $f$ a mapping of $M \cdot Y$ into the $n$-sphere. We may suppose that $f$ has already been extended to a neighborhood $U$ of $M \cdot Y$ and indeed we may suppose that the extension $\bar{f}$ is defined on $\bar{U}$. We may also assume that $Y$ does not lie wholly in $\bar{U}$ and that $F(U)$ meets $Y$. Now $M$ and $Y \cdot F(U)$ are disjoint closed sets. Let $W$ be a neighborhood of $Y \cdot F(U)$ whose closure does not meet the set $M$. Then $P=H-(\bar{W}+(Y-U))$ is an open set containing $M$ since

$$
\begin{aligned}
M \cdot P & =(M-\bar{W}) \cdot((M-Y)+U)) \\
& =(M-Y)+M \cdot U \supset(M-Y)+M \cdot Y \cdot U=M .
\end{aligned}
$$

Now $P$ contains a neighborhood $V$ of $M$ such that $F(V)$ is a $T_{n}$ and we may even suppose that $\bar{V}$ is contained in $P$. Then $Y \cdot F(V)$ is contained in $U$. Let $g=\bar{f} \mid Y \cdot \bar{U} \cdot \bar{V}$. Since $M \cdot Y \subset Y \cdot \bar{U} \cdot \bar{V}$ it follows that $g$ is an extension of $f$. Also

$$
Y=Y \cdot \bar{U} \cdot \bar{V}+(Y-U \cdot V) \text { and } Y \cdot \bar{U} \cdot \bar{V} \cdot(Y-U \cdot V) \subset Y \cdot F(V) .
$$

Since $F(V)$ is a $T_{n}$ the mapping $g \mid Y \cdot F(V)$ may be extended to a transformation $h:(Y-U \cdot V) \rightarrow S_{n}$. Combining $g$ and $h$ we get an extension of $f$ mapping $Y$ into $S_{n}$.

As a corollary to (4.12) we get:

(4.13) If $H$ is a $C_{n}$ then any $T_{n}$-endelement is $a C_{n}$. 
5. Further properties of extension sets. It follows at once from the definition of the sets $J_{n}$ that:

(5.1) For any subset $Z \subset H$ we have $\Delta_{n}(A)=\Delta_{n}(\bar{A})$.

(5.2) If $M$ is a $J_{n}, Z$ a $T_{n}$ and $M-Z=M_{1}+M_{2}$ is a separation then $M_{i}+Z$ is $a J_{n}$.

The proof of this is similar to that of (4.5).

(5.3) If $x$ and $y$ are points of $H$ then neither $x$ nor $y$ is a cut point of $\Delta_{n}(x+y)$ if this set is a continuum.

This is a corollary to (5.2). In this connection see Ayres [1]. Our next result is highly analogous to a well known proposition concerning $A$-sets. Thus, in a Peano space, the complement of each $A$-set is the union of a nullsequence of pair-wise disjoint open sets (the components of its complement) each of which has a $T_{0}$ for its boundary.

(5.4) Let $N$ be a subspace the complement of which is the union of a collection of pair-wise disjoint open sets whose boundaries are sets $T_{n}$. Then $N$ is a $J_{n}$.

Proof. Let $H-N$ be covered by the collection [ $U$ ] of pair-wise disjoint open sets such that $F(U)$ is a $T_{n}$ for each $U \in[U]$. Let $f$ transform $N \cdot Y$ into the $n$-sphere, $Y$ being a subspace. We may extend $f$ to a mapping $g: V \rightarrow S_{n}$ where $V$ is a neighborhood of $Y \cdot N$. In view of the normality of $H$ we can find a neighborhood $W$ of $N \cdot Y$ such that $\bar{W} \subset V$. We have

$$
Y=Y \cdot N+\sum U \cdot Y=Y \cdot W+\sum U \cdot Y .
$$

The sets $Y \cdot W, Y \cdot U$ are open in $Y$ and $Y$ is compact. Hence there is a finite family $U_{1}, U_{2}, \cdots, U_{p}$ such that the sets $Y \cdot W, Y \cdot U_{i}$ cover $Y$. Let $Z_{1}=Y \cdot \bar{W}-U_{1}$ and $Y_{1}=Z_{1}+\bar{U}_{1}$. Then $Z_{1} \cdot \bar{U}_{1}$ is a subset of $Y \cdot \bar{W} \cdot F\left(U_{1}\right)$ and so is a $T_{n}$. The mapping $g \mid Z_{1} \cdot \bar{U}_{1}$ can be extended to a mapping of $\bar{U}_{1}$ into $S_{n}$. We secure in this way a transformation $g_{1}$ of $Z_{1}$ into $S_{n}$ which is an extension of $g$. Let $Z_{2}=Y_{1}-U_{2}$ and $Y_{2}=Z_{2}+\bar{U}_{2}$. Then $Z_{2} \cdot \bar{U}_{2}$ is contained in $Y_{1} \cdot F\left(U_{2}\right)$ and so is a $T_{n}$-set. Accordingly the mapping $g_{1} \mid Z_{2} \cdot \bar{U}_{2}$ may be extended to a transformation $g_{2}$ of $Y_{2}$ into $S_{n}$. By a continuation of this procedure we arrive finally at a mapping $g_{p}: Y_{p} \rightarrow S_{n}$ which is an extension of $g_{p-1}$ and hence of $f$. But $Y$ is a subset of $Y_{p}$ and we are able to infer that $N$ is an extension set.

(5.5) If $X$ is a subspace then each $T_{n}$-endpoint of $\Delta_{n}(X)$ is a $T_{n}$-endpoint of $X$.

Proof. There is no loss of generality if we let $H$ be the set $\Delta_{n}(X)$. Thus let $p$ be a $T_{n}$-endpoint of $H$ not in $X$ so that there is a neighborhood $U$ containing $p$ which does not meet $X$. Then $U$ contains a neighborhood $V$ of $p$ 
for which $F(V)$ is a $T_{n}$-set. But then $H-V$ is a $J_{n}$ (by (5.4)) which contains $X$ but not $p$. This is a contradiction.

If we regard the sets $J_{n}$ as "closed" sets then (5.5) states that any subspace "dense" in $H$ contains all $T_{n}$-endpoints. In this connection see Ayres [1]. We remark that (5.5) remains valid if " $T_{n}$-endpoint" is replaced by " $T_{n}$-endelement."

(5.6) If $M+N$ and $M \cdot N$ are $J_{n}$-sets then so also are $M$ and $N$ if they are subspaces.

Proof. Let $Y$ be a subspace and let $f$ be a mapping of $Y \cdot M$ into the $n$ sphere. The cases in which $Y \cdot N=0$ or $Y \cdot M \cdot N=0$ are readily treated. The only other case is that in which $Y \cdot M \cdot N$ is not null. Then $f \mid Y \cdot M \cdot N$ can be extended to a mapping $g$ of $Y \cdot N$ into $S_{n}$. Call this mapping $g$. If we combine $f$ and $g$ we get a transformation $h: Y \cdot(M+N) \rightarrow S_{n}$. This mapping may be extended to $Y$ by our hypothesis.

(5.7) If $X$ is a $C_{0}$ then $\Delta(X)$ is a $C_{0}$.

Proof. For if not, then we would have a separation as in (5.2) with $Z=0$. If $X$ is a $C_{0}$ it must lie in one of the sets $M_{1}$ or $M_{2}$, say $M_{1}$. But $M_{1}$ is a $J_{n}$ by (5.2).

(5.8) Let $Z$ be the set of all points of $H$ that are not $T_{n}$-endpoints. Then, if $H$ is a $C_{n}$, the set $Z$ is n-connected.

Proof. If not let $f: Z \rightarrow S_{n}$ which is essential on the subspace $X$. Since $H$ is an $n$-continuum and thus $f$ cannot be extended to $H$ it follows by (2.6) that $f$ admits an essential membrane $Y$. Suppose that the point $p$ of $Y$ is a $T_{n}$-endpoint. Then there exists a neighborhood $V$ of $p$ such that $F(V)$ is a $T_{n}$ and $V \subset H-X$, since $X$ is closed and $p$ is not in $X$. Now $X \subset Y-V$ and since $Y$ is an essential membrane $f$ can be extended to $\bar{f}:(Y-V) \rightarrow S_{n}$. Since $Y=Y \cdot \bar{V}+(Y-V)$ and $Y \cdot \bar{V} \cdot(Y-V)$ is a $T_{n}$ it follows that $\bar{f} \mid Y \cdot F(V)$ can be extended to $Y \cdot \bar{V}$. Thus $f$ can be extended to $Y$, a contradiction. Hence $Y$ must be contained in $Z$ and so $f$ is defined on $Y$. This is impossible. Hence $S$ is $n$-connected. For $n=0$ this result is well known.

\section{BiBLIOGRAPHY}

1. W. L. Ayres, Concerning the arc-curves and basic sets of a continuous curve, Trans. Amer. Math. Soc. vol. 30 (1928) pp. 567-578.

2. S. Eilenberg, Sur les transformations d'espaces metriques en circonference, Fund. Math. vol. 24 (1935) pp. 160-176.

3. W. Hurewicz and H. Wallman, Dimension theory, Princeton Mathematical Series, vol. 4, 1941.

4. S. Lefschetz, Topics in topology, Annals of Mathematics Studies, no. 10, 1942.

5. L. Lusternik and L. Schnirelmann, Methodes topologiques, Paris, 1934. 
6. J. Rozanska, Über stetige Abbildungen eines Elementes, Fund. Math. vol. 28 (1937) pp. 219-232.

7. A. D. Wallace, Monotone transformations, Duke Math. J. vol. 9 (1942) pp. 487-506.

8. G. T. Whyburn, Cyclic elements of higher order, Amer. J. Math. vol. 56 (1934) pp. 133146.

9. - Analytic topology, Amer. Math. Soc. Colloquium Publications, vol. 28, 1942.

10. R. L. Wilder, Point sets in three and higher dimensions and their investigation by means of a unified analysis situs, Bull. Amer. Math. Soc. vol. 38 (1932) pp. 649-692.

11. - The sphere in topology, Amer. Math. Soc. Semicentennial Publications, vol. 2, 1938, pp. 136-184.

12. C. Borsuk, Über Rand- und Kernpunkte kompakter metrischer Räume, Ergebnisse eines mathematischen Kolloquiums, Vienna, 1933, pp. 28-32.

13. H. Hopf, Über die Abbildungen von Sphären, Fund. Math. vol. 25 (1935) pp. 427-440.

Tine University of Pennsylvania,

Philadelphia, Pa. 